\title{
Um Relato de Experiência da Escola de Verão de Programação para Crianças
}

\author{
Lara Araujo, Julia Godinho, Janaína Gomide \\ ${ }^{1}$ Universidade Federal do Rio de Janeiro (UFRJ) - Campus Macaé \\ Macaé - RJ - Brasil \\ \{araujo.lara15, juliagodinho08\}@gmail.com, janainagomide@macae.ufrj.br
}

\begin{abstract}
The goal of the Summer School of Programming was offering a fun and interactive environment of learning programming during school vacation. This work proposes to contribute as support material to projects and schools that want to reproduce this method in other environments. This report contemplates the process used in the classroom and the results obtained. The structure of the classes were standardized and followed the following process: theoretical introduction, dynamics, programming and revision. Previous to the course, $70 \%$ of students did not know what programming was. In the end, 100\% of the children rated their experience as very good or good and, through a questionnaire, it was found that they had understood the subjects given.
\end{abstract}

Resumo. A Escola de Verão de Programação teve como objetivo oferecer um ambiente divertido e interativo de ensino de programação nas férias escolares. A experiência relatada neste trabalho propõe contribuir como material de apoio a projetos e escolas que queiram reproduzir este método em outros ambientes. Este relato contempla o processo utilizado em sala de aula e os resultados obtidos. A estrutura das aulas foram padronizadas e seguiam o seguinte processo: introdução teórica, dinâmica, programação e revisão. Anteriormente ao curso, $70 \%$ dos alunos não sabiam o que era o programação. Ao final, $100 \%$ das crianças classificaram sua experiência como muito boa ou boa e, por meio de um questionário, constatou-se que haviam compreendido os temas ministrados.

\section{Introdução}

As habilidades adquiridas com a programação vão além de criar jogos, websites e sistemas. Desenvolve também a capacidade de resolução de problemas, criatividade, trabalho em equipe e organização de pensamento e ideias [Wing 2006]. É possível aproveitar a aproximação das crianças com os meios eletrônicos para incentivá-las a criarem seus próprios jogos e aplicativos, transformando-as em desenvolvedoras de soluções tecnológicas.

Há diversas iniciativas de ensino de programação e robótica para crianças e jovens [Aono et al. 2017, Garcia et al. 2016, Rodrigues et al. 2016, Souza and Mombach 2016, von Wangenheim et al. 2017, Godinho et al. 2017, Gomes et al. 2016, Scaico et al. 2013]. Essas iniciativas, tanto no Brasil como nos Estados Unidos, envolvem cursos em escolas no ensino fundamental e médio, durante o período em que esse público se encontra em sala de aula.

Além do uso de ferramentas digitais, outra estratégia utilizada para promover a disseminação do pensamento computacional são atividades lúdicas, sem o uso de computadores. [Bell et al. 2005] apresenta a metodologia da Computação Desplugada, a 
VII Congresso Brasileiro de Informática na Educação (CBIE 2018)

Anais do XXIV Workshop de Informática na Escola (WIE 2018)

qual destina-se ao ensino de princípios de programação sem a necessidade de hardware e/ou software especializados para essa tarefa. Os relatos de [Costa et al. 2012] e [França et al. 2012] evidenciam a aplicação desse método no ensino da Computação.

Acampamentos de verão de programação são oferecidos há décadas nos Estados Unidos de acordo com [DiDio 2017]. Estima-se que mais de 22 mil estudantes participaram dos 95 cursos de verão oferecidos somente no ano de 2017. Nesse cenário, observouse a oportunidade de lecionar no Brasil a programação fora do ambiente escolar para que as crianças se sentissem mais entusiasmadas em aprender esse conteúdo.

Sobre essa perspectiva de ensino lúdico, professores experientes são unânimes em dizer que esse método corrobora com o desenvolvimento da criança, torna o aprendizado mais prazeroso, além de facilitar o trabalho do professor [Oliveira 2009]. Nesse sentido, essa pesquisa teve o intuito de propor a Escola de Verão de Programação para crianças, em período de férias escolares, baseada em métodos lúdicos com jogos e computação desplugada. Além disso, esse trabalho expõe a experiência obtida nessa escola sob a perspectiva dos monitores e os alunos participantes. Desta forma, este artigo contribui como sugestão de material de apoio para escolas e projetos que tenham interesse em organizar eventos similares.

\section{Perfil dos Alunos}

Participaram da Escola de Verão de Programação 58 crianças, sendo 42 meninos e 16 meninas, entre cinco e doze anos. Mais de $50 \%$ dos alunos estudam na rede pública de ensino de Macaé e região. Verificou-se que a maioria dos alunos não utiliza o computador no seu dia a dia e aproximadamente $58 \%$ utilizam menos de uma hora por semana para fins educacionais. Para a maioria dos alunos aprender programação de computadores era algo novo. Aproximadamente $90 \%$ deles não sabiam como era feito um programa de computador e mais de $68 \%$ deles responderam que não conheciam sobre Ciência da Computação.

\section{Estratégia de Ensino Lúdico}

Esta seção descreve a estrutura padronizada criada para cada aula e o plano de aulas utilizado na Escola de Verão de Programação com detalhes sobre todas as atividades desenvolvidas. Definiu-se uma estrutura padrão a ser utilizada em todas as aulas de forma que as crianças tivessem contato com um novo princípio da programação em cada aula. O processo adotado foi dividido em quatro momentos (Figura 1).

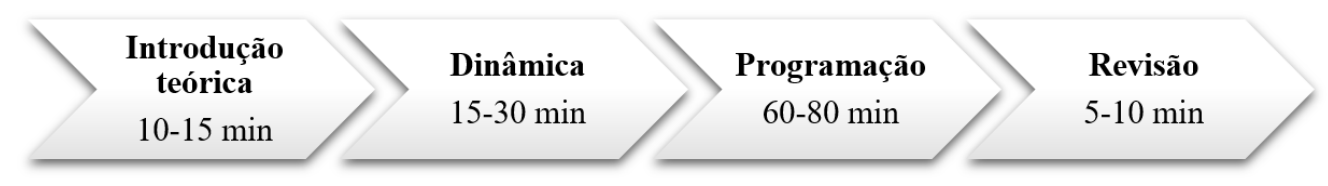

Figura 1. Estrutura padrão criada para todas as aulas.

A aula se inicia com uma Explicação teórica sobre um conceito de programação, em seguida é realizada uma atividade de Computação Desplugada envolvendo os alunos e mostrando o conceito teórico dentro de suas realidades. Após, inicia-se a Programação 
VII Congresso Brasileiro de Informática na Educação (CBIE 2018)

Anais do XXIV Workshop de Informática na Escola (WIE 2018)

Tabela 1. Plano de aulas da Escola de Verão de Programação

\begin{tabular}{|c|c|c|c|}
\hline Aula & Conceito & Dinâmica & Ferramenta \\
\hline 1 & Algoritmo & "Descreva o Objeto" e "Barco de Papel" & Code Studio \\
\hline 2 & Repetição & "Seguindo Instruções" & Scratch \\
\hline 3 & Condicional e Variável & $\begin{array}{l}\text { "Condicionais com Cartas" e "Variáveis } \\
\text { em Envelopes" }\end{array}$ & Scratch \\
\hline 4 & Booleano e Binário & $\begin{array}{l}\text { "Contando os Pontos" e "Braceletes de } \\
\text { Binário" }\end{array}$ & Monster Coding \\
\hline 5 & Revisão & "Guie a Tartaruga" & Pencil Code \\
\hline 6 & Revisão & Revisão interativa & CodeMonkey \\
\hline
\end{tabular}

de Computadores e os alunos são levados ao computador para aplicar o conceito visto com o auxílio de uma ferramenta online. A aula é finalizada com uma Revisão sobre o assunto.

A Escola de Verão de Programação foi realizada ao longo de seis encontros, cada um com duração de duas horas. Os instrutores do curso são graduandos da Universidade Federal do Rio de Janeiro e participam do projeto de extensão Aprenda a Programar Jogando $^{1}$, que ensina jovens e crianças a programarem. Participaram do curso 58 crianças e 10 instrutores. As crianças foram divididas em turmas de até doze alunos. O curso ocorreu durante as férias do primeiro semestre de 2018 nos laboratórios de informática, os quais totalizam 35 computadores com acesso à internet.

A Tabela 1 apresenta o plano de aulas utilizado. Os conceitos escolhidos para serem abordados nesse curso fazem parte das competências e habilidades relacionadas ao ensino da Computação para as etapas de Educação Básica apresentadas em [Raabe et al. 2017]. As ferramentas utilizadas são gratuitas e disponíveis na Web. Elas promovem um ambiente de programação direcionado a crianças e jovens de forma intuitiva e divertida por meio da linguagem de programação em blocos ou textual. A seguir cada encontro é descrito em detalhes apresentando as dinâmicas realizadas, os materiais complementares necessários e as atividades realizadas em cada ferramenta.

\subsection{Aula 1: Algoritmo e Code Studio}

A primeira aula introduz o conceito de algoritmo como uma sequência simples de instruções. Para tal, foram escolhidas atividades, nas quais os alunos são encorajados a criar algoritmos para situações cotidianas, usando comandos com clareza e objetividade.

Foram realizadas duas dinâmicas. A primeira foi "Descreva o objeto", uma variação da atividade Marching Orders disponível em [Bell et al. 2005]. Para isso, as crianças escolhem uma imagem e dão comandos orientando o professor a desenha-la corretamente. Durante a dinâmica "Barco de papel", uma adaptação da dinâmica Real-Life Algorithms: Paper Planes disponível em [Code.org 2017], os alunos ordenam corretamente um conjunto de comandos dados, a fim de formarem um passo a passo de montagem de um barco de papel.

\footnotetext{
${ }^{1}$ Site: aprendaprogramar.macae.ufrj.br
} 
VII Congresso Brasileiro de Informática na Educação (CBIE 2018)

Anais do XXIV Workshop de Informática na Escola (WIE 2018)

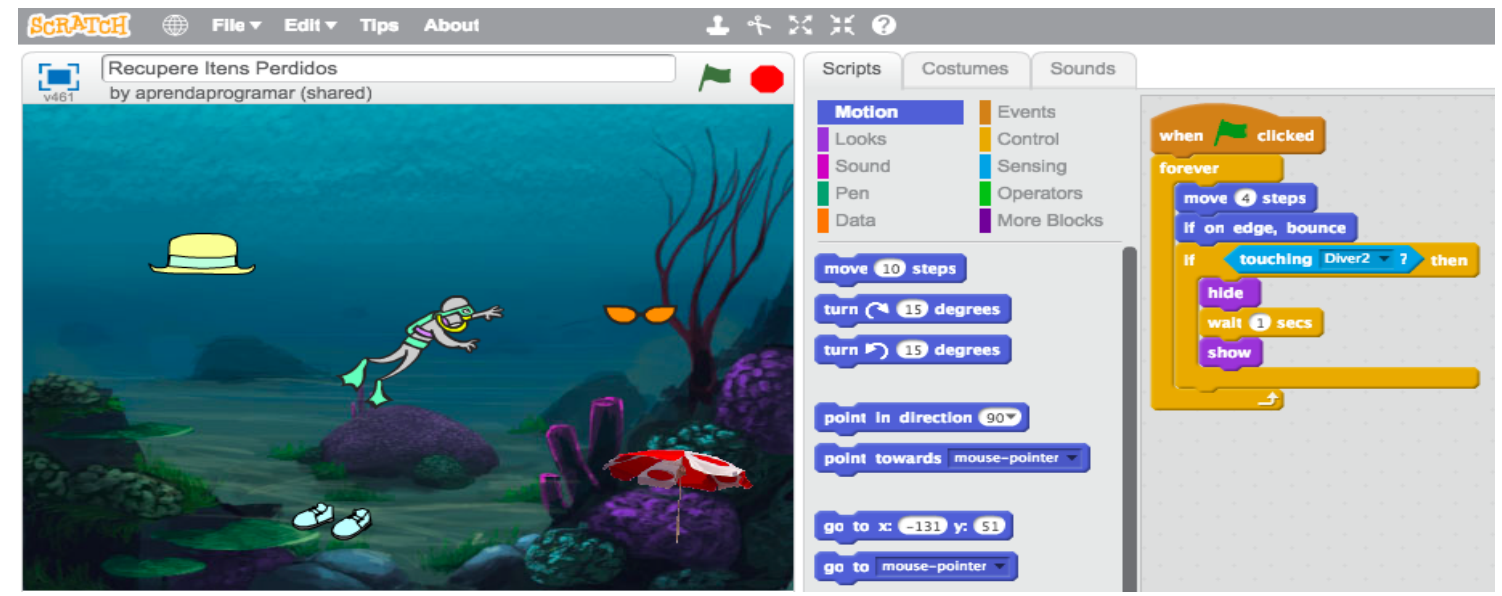

Figura 2. Atividade de programação "Recupere Itens Perdidos" na ferramenta Scratch.

A ferramenta utilizada na primeira aula foi Code Studio $^{2}$ que reune uma série de atividades com objetivo de apresentar conceitos de programação para o público infantil. A maioria dessas atividades utilizam a programação Blockly, na qual os comandos são apresentados em blocos gráficos que se encaixam para formar um código. Dentre as atividades disponíveis foram utilizados tutoriais autoguiados com temas conhecidos como Frozen, Minecraft e Star Wars. Essas atividades são promovidas pela organização Code.org e fazem parte de um movimento global no qual oferece tutoriais de uma hora ${ }^{3}$. As crianças puderam escolher o tema que mais se interessavam para realizar a atividade com duração de uma hora.

\subsection{Aula 2: Movimentos, Repetição e Scratch}

A aula 2 destaca o uso de comandos de repetição com o auxílio da dinâmica "Seguindo Instruções", uma adaptação da atividade Marching Orders disponível em [Bell et al. 2005]. A turma deve dar comandos a um aluno vendado, como: "Andar", "Virar à esquerda" e "Virar à direita", a fim de que ele consiga completar um circuito. Depois de realizar o percurso uma vez o instrutor repete a dinâmica. Na segunda vez, ele altera o percurso e introduz um novo comando, "Repete", e as crianças aprendem quando usa-lo corretamente.

Esses conceitos são praticados na ferramenta $S$ cratch ${ }^{4}$ que utiliza a programação em blocos. Essa ferramenta permite que a criança crie uma animação ou jogo de forma livre e estimula a criatividade e curiosidade. A atividade proposta se chama "Recupere Itens Perdidos" (Figura 2) e foi preparada para que os alunos possam aplicar os conceitos de movimento e loops. Nessa atividade, a criança deve criar um cenário (fundo do mar) e adicionar os personagens (mergulhador e outros objetos). O mergulhador deve ser programado para seguir o cursor do mouse enquanto os objetos se movem de forma aleatória no jogo. O objetivo é que o mergulhador alcance os objetos, os resgatando.

\footnotetext{
${ }^{2}$ Disponível em: code.org/

${ }^{3}$ Disponível em: https://hourofcode.org/

${ }^{4}$ Disponível em: https://scratch.mit.edu/
} 
Se (CARTA é VERMELHA)

SEU time ganha 1 ponto

Senão OUTRO time ganha 1 ponto

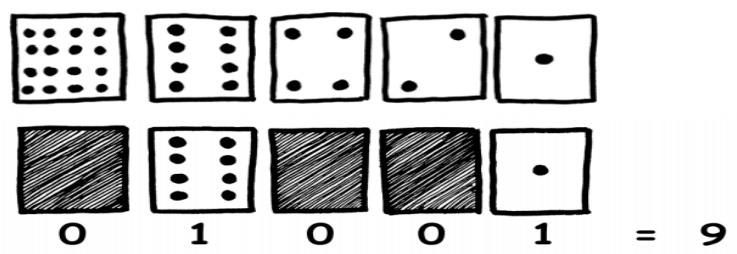

Figura 4. Cartas da dinâmica "Contando os Pontos". Fonte: [Bell et al. 2005].

Figura 3. Carta de regra da dinâmica "Condicionais com Cartas".

\subsection{Aula 3: Eventos, Condicional, Variável e Scratch}

Apesar de comandos condicionais terem sido abordados em aulas anteriores, a aula 3 é dedicada a ressaltar a importância do uso das estruturas de condição e introduzir o conceito de variável. Duas dinâmicas são realizadas. A primeira dinâmica, "Condicionais com Cartas", é uma adaptação da atividade If/Else: Conditionals with Cards disponível em [Code.org 2017]. Baseado no pseudocódigo dado pelo instrutor, conforme a Figura 3, os alunos decidem a distribuição dos pontos a cada carta virada no topo do baralho.

A segunda dinâmica é a "Variável em envelopes", adaptada da atividade Envelope Variables disponível em [Code.org 2017]. Essa prática aborda as variáveis como uma forma de armazenar as informações. A turma é dividida em alguns grupos e cada um recebe envelopes com as inscrições "NOME" ou "IDADE" e papéis onde as informações devem ser escritas pelas crianças e colocadas dentro dos envelopes. Os alunos usam os envelopes para completar as lacunas de uma frase. Por exemplo, na frase "Eu me chamo NOME e tenho IDADE anos", os envelopes com os respectivos títulos devem ser colocados e o conteúdo do envelope deve ser revelado para completar as informações "NOME" e "IDADE" da frase.

Após compreenderem o conceito de variável, as crianças vão continuar a programação do jogo "Recupere Itens Perdidos" na ferramenta Scratch, complementando-o. Foram adicionadas variáveis para armazenar os pontos por cada item recuperado e uma condição que finalizasse o jogo.

\subsection{Aula 4: Valores Booleanos, Binário e Monster Coding}

O conceito de programação dessa aula são valores booleanos. Os alunos aprendem sobre verdadeiro e falso, que são relacionados com valores binários. Para abordar esses fundamentos são implementadas duas dinâmicas. A primeira, "Contando os Pontos", visa ensinar como a transformação dos números decimais para binários baseada em Count the Dots-Binary Numbers disponível em [Bell et al. 2005]. O único material necessário é um conjunto de cartas mostrado na Figura 4.

Logo após serem introduzidos ao sistema binário, os alunos se divertem com a dinâmica baseada na atividade Binary Bracelets do [Code.org 2017]. Com uma tabela do alfabeto em código binário, os alunos criam braceletes com as iniciais dos seus nomes, usando miçangas correspondentes aos números " 1 " e "0" do sistema binário.

A ferramenta utilizada nessa aula foi a Monster Coding ${ }^{5}$. A versão utilizada dessa plataforma foi autoguiada e as crianças juntam os blocos de comandos para completar

\footnotetext{
${ }^{5}$ Disponível em: http://monstercoding.com/
} 
VII Congresso Brasileiro de Informática na Educação (CBIE 2018)

Anais do XXIV Workshop de Informática na Escola (WIE 2018)

as missões. O personagem principal dessa ferramenta é um monstro que precisa realizar algumas tarefas para sair da ilha. Por meio de perguntas, os conceitos de programação vistos anteriormente são revisados ao longo do tutorial.

\subsection{Aula 5: Revisão e Pencil Code}

A quinta aula se iniciou com uma revisão de todos os conceitos com um jogo de perguntas e respostas. A dinâmica dessa aula foi elaborada pelos instrutores do curso e intitulada "Guie a tartaruga". A dinâmica consiste em utilizar uma caneta de quadro branco colada à imagem de uma tartaruga e um aluno voluntário usa a caneta, seguindo comandos dados por seus colegas, para formar figuras escolhidas previamente pelo professor. O objetivo é que os alunos construam algoritmos para desenhar objetos e, além disso, entendam o funcionamento da ferramente que será utilizada posteriormente.

A ferramenta dessa aula é o Pencil Code ${ }^{6}$. Essa plataforma é de uso livre e não apresenta um tutorial a ser seguido. Nessa ferramenta os alunos podem alternar livremente entre a programação de blocos e escrita. O personagem principal dessa plataforma é uma tartaruga que deve ser programada para seguir comandos e realizar desenhos na tela. Os alunos aprenderam a desenhar um quadrado e uma escada usando comandos "Para frente", "Direita", "Esquerda", "Repete". Além disso, foram incentivados a usarem a criatividade e criarem outras formas e objetos.

\subsection{Aula 6: Revisão e CodeMonkey}

$\mathrm{Na}$ última aula os conceitos de programação são novamente revisados. A aula inicia-se com uma revisão interativa na qual os conceitos de programação são escritos no quadro e os próprios alunos escolhem um tema para explicarem ao restante dos colegas.

A plataforma online dessa aula é a CodeMonkey ${ }^{7}$ na qual utiliza exclusivamente programação escrita. A versão utilizada foi a Hour of Code que é um tutorial autoguiado com duração de uma hora. O principal personagem dessa ferramenta é um macaco que segue o código escrito pelo usuário em cada missão. São abordados comandos de movimento e repetição na linguagem de programação CoffeeScript.

Ao final da aula, aplica-se um questionário para as crianças, com objetivo de verificar o nível de interesse, aprendizado e dificuldades encontradas durante o curso.

\section{Relato de Experiência dos Monitores e Alunos}

Nesta seção são apresentados os relatos de experiência dos monitores que conduziram as turmas da Escola de Verão de Programação, o relato das crianças participantes e uma análise das questões de conhecimento respondidas por eles.

\subsection{Relato dos Monitores}

Ao final do curso, um questionário sobre a Escola de Verão foi respondido pelos instrutores. As questões contidas tinham a finalidade de entender como foi a experiência e analisar diferentes etapas da Escola de Verão sob a perspectiva dos monitores.

\footnotetext{
${ }^{6}$ Disponível em: https://pencilcode.net/

${ }^{7}$ Disponível em: https://www.playcodemonkey.com/
} 
VII Congresso Brasileiro de Informática na Educação (CBIE 2018)

Anais do XXIV Workshop de Informática na Escola (WIE 2018)

Os instrutores relataram ter sido uma excelente experiência na qual puderam compartilhar conhecimento entre si e também com os alunos. Além disso, noticiaram poder acompanhar o desenvolvimento dos alunos e proporcionar a estudantes que, no geral, não têm acesso a computação, uma oportunidade de aprender sobre programação.

O desempenho dos alunos também pôde ser analisado pela observação dos monitores. Foi verificado que o uso da metodologia de Computação Desplugada no início das aulas teve grande nível de aceitação por parte dos alunos e favoreceu a compreensão dos conceitos de programação por meio de associações com o cotidiano.

Os maiores desafios encontrados pelos monitores durante as aulas foram referentes a problemas com atenção e concentração dos alunos. Esses problemas foram minimizados encontrando abordagens diferentes e mais interativas para passar o conteúdo e manter a atenção deles. Outro problema evidenciado foi relacionado a crianças não alfabetizadas, pois durante a maior parte das atividades era necessário a leitura de comandos em blocos ou tarefas. Nesse caso, foi necessário o acompanhamento individual de um monitor.

No que se refere a duração de aulas, os monitores relataram que a quantidade total de seis encontros foi suficiente para introduzir os conceitos básicos e apresentar as plataformas de programação online. A frequência de duas vezes na semana também foi considerada suficiente para manter o contato da criança com a programação e não sobrecarregá-la. Entretanto, foi ressaltado que com mais aulas o conteúdo poderia ser melhor trabalhado.

Quando os monitores foram perguntados sobre "Qual tema gerou maior dificuldade nos alunos? Por quê?", as respostas ficaram divididas entre condicionais, variáveis e valores booleanos. E na pergunta "Qual tema foi melhor compreendido pelos alunos? Por quê?", os monitores falaram que foram os conceitos de algoritmo, movimento e repetição.

Sob a perspectiva dos monitores, as maiores dificuldades encontradas pelos alunos foram na compreensão e uso dos conceitos de valores booleanos, condicionais e variáveis. Enquanto que os melhores compreendidos por eles foram algoritmos, movimento e repetição.

\subsection{Relato e Avaliação dos Alunos}

Para obter o relato da experiência das crianças que participaram da Escola de Verão de Programação, foi aplicado um questionário que compreende o nível de satisfação, aprendizado e dificuldade. Algumas perguntas foram baseadas no questionário realizado em [Duarte et al. 2017]. Das cinquenta e oito crianças que estavam presentes no primeiro dia, quarenta finalizaram o curso e $100 \%$ desses alunos responderam que gostaram muito ou que gostaram de terem participado.

A ferramenta escolhida como favorita pelos alunos foi a Scratch e a que eles menos gostaram foi a Monster Coding. Apesar dessas escolhas, todas as ferramentas receberam avaliações positivas de pelo menos $80 \%$ dos alunos. Em relação ao nível de dificuldade, Pencil Code foi a que obteve um maior percentual de respostas como "fácil"; como "médio", Monster Coding e Scratch ficaram empatados; e a ferramenta que mais pessoas classificaram como "difícil" foi Code Monkey.

Para verificar o aprendizado dos alunos foram realizadas três perguntas que estão 
VII Congresso Brasileiro de Informática na Educação (CBIE 2018)

Anais do XXIV Workshop de Informática na Escola (WIE 2018)

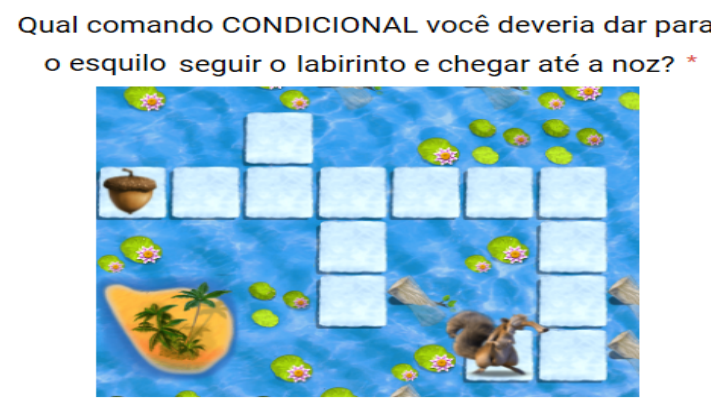

Figura 5. Desafio envolvendo comando condicional.
Qual comando de REPETIÇÃO (LOOP) usar para a tartaruga formar a figura abaixo?

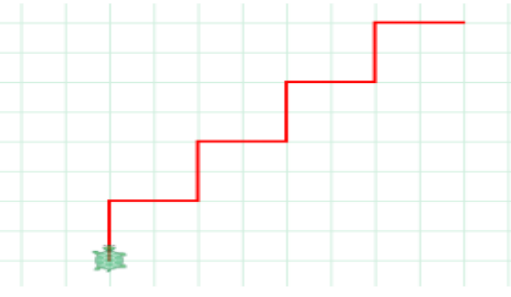

Figura 6. Desafio envolvendo comando de repetição.

apresentados na Tabela 2 juntamente com as respostas e a porcentagem de acertos. As respostas foram avaliadas considerando a linguagem informal e as associações com o cotidiano e as dinâmicas feitas dentro de sala para facilitar o entendimento. Pode-se constatar que a maioria dos alunos acertou todas as questões e compreendeu os conceitos de algoritmos, valor booleano e variável.

Tabela 2. Perguntas discursivas para verificar o aprendizado dos conceitos de programação, respostas mais frequentes e porcentagem de acertos

\begin{tabular}{|l|l|l|}
\hline Perguntas & Respostas mais frequentes & Acertos \\
\hline 1. O que é algoritmo? & $\begin{array}{l}\text { "passo a passo" } \\
\text { "ordem de comandos" } \\
\text { "receita" }\end{array}$ & $55,26 \%$ \\
\hline 2. Quais são os valores booleanos? & $\begin{array}{l}\text { "verdadeiro e falso" } \\
\text { "verdade e mentira" } \\
\text { "0 e 1" }\end{array}$ & $76,32 \%$ \\
\hline 3. O que é uma variável? & $\begin{array}{l}\text { "caixinha" } \\
\text { "armazenamento" } \\
\text { "pontos" } \\
\text { "variação de dados" }\end{array}$ & $60,53 \%$ \\
\hline
\end{tabular}

Além das questões discursivas, dois desafios de múltipla escolha estavam presentes na avaliação (Figuras 5 e 6). Foram disponibilizadas quatro opções de resposta para cada pergunta, sendo somente uma correta.

Em relação ao desafio de condicional, 42,5\% acertaram completamente, 37,5\% erraram a condição do comando condicional e $20 \%$ não usaram uma estrutura condicional. Já considerando o desafio de repetição, 35\% acertaram completamente, $25 \%$ erraram o número de repetições e $40 \%$ erraram a sequência dos comandos.

Quando perguntados "O que você mais gostou nas aulas?", algumas das respostas foram: "computação e as dinâmicas", "os jogos e conversas", "dos computadores, dos amigos e dos jogos", "de programar jogos", "de todas as coisas", entre outras. Pode-se observar que os alunos ficaram muito alegres com o contato com o computador, gostaram muito de terem conhecido os jogos usados para ensinar programação e ficaram contentes por, além de adquirir conhecimento, conhecer novas pessoas durante as férias escolares. 
VII Congresso Brasileiro de Informática na Educação (CBIE 2018)

Anais do XXIV Workshop de Informática na Escola (WIE 2018)

Durante as aulas os monitores relataram o entusiasmo das crianças em aprender novos conceitos, descobrir novas funcionalidades e desenvolver seus próprios jogos. Alguns depoimentos nos questionários confirmam esse fato, como: "Eu acho muito divertido, interativo e também é legal fazer novos amigos. A gente aprende bastante coisa." (9 anos); "Estou achando legal fazer jogos. Depois podemos até trabalhar com isso" (10 anos); "Estou adorando, porque é o que quero fazer. Quero ser cientista" (8 anos).

No geral, alunos sem nenhum conhecimento prévio de programação conseguiram completar as atividades propostas, descobrindo as funcionalidades das ferramentas, superando as dificuldades e aprendendo a solucionar os problemas encontrados durante $o$ desenvolvimento dos programas.

\section{Conclusão}

Este artigo apresentou as atividades realizadas durante a Escola de Verão de Programação, cujo objetivo foi introduzir os conceitos básicos de programação de forma prática e divertida para crianças. No total, 58 crianças entre 5 e 12 anos participaram de atividades de programação durante três semanas no período das férias escolares.

As aulas seguiram uma estrutura padrão que abrange uma explicação teórica, a realização de atividades de Computação Desplugada, o uso de ferramenta de programação e uma breve revisão. Essa estrutura foi planejada visando praticar de forma lúdica um novo conceito de programação a cada aula. A experiência relatada neste trabalho propõe uma sugestão de material de apoio para os que quiserem organizar eventos similares.

De todas as crianças que participaram, a maioria não usa o computador no seu dia a dia e $100 \%$ dos alunos não sabia como um programa de computador é feito. Porém, ao final do curso, $85 \%$ dos alunos disseram ter interesse em aprender mais sobre Ciência da Computação. Além disso, a maioria dos alunos acertou as perguntas sobre os conceitos de programação e as respostas estavam associadas às dinâmicas realizadas. A maioria dos participantes disse ter gostado muito das atividades e houveram relatos de alunos que gostariam de ter mais aulas e aprender mais sobre computadores e programação.

De acordo com o relato dos monitores, observa-se que o plano de aulas foi estruturado de forma adequada, pois possibilitou que os alunos se divertissem enquanto aprendiam de forma efetiva o conteúdo. Dentre os trabalhos futuros, planeja-se elaborar outras formas de avaliação dos alunos para verificar a aplicação dos conceitos ensinados.

\section{Referências}

Aono, A. H., Rody, H. V. S., Musa1, D. L., Pereira1, V. A., and Almeida, J. (2017). A utilização do scratch como ferramenta no ensino de pensamento computacional para crianças. In XXXVII Congresso da Sociedade Brasileira de Computação - $25^{\circ}$ Workshop sobre Educação em Computação (WEI), pages 2169-2178.

Bell, T. C., Witten, I. H., Fellows, M. R., Adams, R., and McKenzie, J. (2005). CS Unplugged: An enrichment and extension programme for primary-aged students. Computer Science Unplugged, Canterbury, New Zealand.

Code.org (2017). CS fundamentals unplugged. https://code.org/curriculum/unplugged.

Costa, T., Batista, A., Maia, M., Almeida, L., and Farias, A. (2012). Trabalhando fundamentos de computação no nível fundamental: experiência de licenciandos em 
VII Congresso Brasileiro de Informática na Educação (CBIE 2018)

Anais do XXIV Workshop de Informática na Escola (WIE 2018)

computação da universidade federal da paraíba. In XX Workshop de Educação em Computação-WEI. Curitiba, PR, Brasil.

DiDio, L. (2017). The value of free stem and coding bootcamps. ACM News, Communications of the ACM.

Duarte, K. T. N., Silveira, T. R. S., and Borges, M. A. F. (2017). Abordagem para o ensino da lógica de programação em escolas do ensino fundamental ii através da ferramenta scratch 2.0. In VI Congresso Brasileiro de Informática na Educação (CBIE 2017) Anais do XXIII Workshop de Informática na Escola (WIE 2017), pages 175-184.

França, R. d., Silva, W. d., and Amaral, H. d. (2012). Ensino de ciência da computaçao na educaçao básica: Experiências, desafios e possibilidades. In $X X$ Workshop sobre Educação em Computação.

Garcia, V. R., da Silva, D. O., de Oliveira, I. G., Trindade, D. d. F. G., Sgarbi, E. M., and do Nascimento, L. F. L. (2016). Despertando jovens talentos com o conhecimento da computação. In Anais do Workshop de Informática na Escola, volume 22, page 583.

Godinho, J., Torres, K., Batista, G., Andrade, E., and Gomide, J. (2017). Projeto aprenda a programar jogando: Divulgando a programação de computadores para crianças e jovens. In XXXVII Congresso da Sociedade Brasileira de Computação - $25^{\circ}$ Workshop sobre Educação em Computação (WEI), pages 2140-2149.

Gomes, T., Melo, J., and Tedesco, P. (2016). Jogos digitais no ensino de conceitos de programação para crianças. In Brazilian Symposium on Computers in Education (Simpósio Brasileiro de Informática na Educação-SBIE), volume 27, page 470.

Oliveira, M. L. (2009). (Im)pertinências da educação: o trabalho educativo em pesquisa. Editora UNESP.

Raabe, A. L. A. et al. (2017). Referenciais de formação em computação: Educação básica. In Sociedade Brasileira de Computação.

Rodrigues, L. C., Queiroga, A., Oliveira, M., and More, A. (2016). Relato de experiência: curso de introdução à programação para crianças do ensino fundamental no ifsp votuporanga. In Anais do Workshop de Informática na Escola, volume 22, page 349.

Scaico, P. D., de Lima, A. A., Azevedo, S., da Silva, J. B. B., Raposo, E. H., Alencar, Y., Mendes, J. P., Scaico, A., et al. (2013). Ensino de programação no ensino médio: Uma abordagem orientada ao design com a linguagem scratch. Revista Brasileira de Informática na Educação, 21(02):92.

Souza, P. S. and Mombach, J. (2016). Ensino de programação para crianças através de práticas colaborativas nas escolas. In Anais do Workshop de Informática na Escola, volume 22, page 545 .

von Wangenheim, C. G., Alves, N. C., Rodrigues, P. E., and Hauck, J. C. (2017). Teaching computing in a multidisciplinary way in social studies classes in school-a case study. International Journal of Computer Science Education in Schools, 1(2).

Wing, J. M. (2006). Computational thinking. Communications of the ACM, 49(3):33-35. 